Preprint typeset in JHEP style - HYPER VERSION

\title{
DUALITY TWISTS ON A GROUP MANIFOLD
}

\author{
Aybike Çatal-Özer \\ School of Mathematics, Trinity College Dublin \\ Dublin 2 IRELAND \\ e-mail: aybike@maths.tcd.ie
}

\begin{abstract}
We study duality-twisted dimensional reductions on a group manifold $\mathrm{G}$, where the twist is in a group $\tilde{G}$ and examine the conditions for consistency. We find that if the duality twist is introduced through a group element $\tilde{g}$ in $\tilde{G}$, then the flat $\tilde{G}$-connection $A=\tilde{g}^{-1} d \tilde{g}$ must have constant components $M_{n}$ with respect to the basis 1 -forms on $G$, so that the dependence on the internal coordinates cancels out in the lower dimensional theory. This condition can be satisfied if and only if $M_{n}$ forms a representation of the Lie algebra of $G$, which then ensures that the lower dimensional gauge algebra closes. We find the form of this gauge algebra and compare it to that arising from flux compactifications on twisted tori. As an example of our construction, we find a new five dimensional gauged, massive supergravity theory by dimensionally reducing the eight dimensional Type II supergravity on a three dimensional unimodular, non-semi-simple, non-abelian group manifold with an $S L(3, \mathbb{R})$ twist.
\end{abstract}




\section{Contents}

1. Introduction

2. Dimensional Reduction on a Group Manifold

3. Twisted Reductions on a Group Manifold 5

3.1 Action 6

3.2 Gauge Algebra

4. Three Dimensional Group Manifolds and Twisted Tori 10

5. Example 14

6. Outlook 17

A. Dimensional Reduction of $\mathcal{L}_{8}$

\section{Introduction}

In the toroidal dimensional reduction of a theory invariant under a global symmetry group $\mathrm{G}$, it is possible to introduce a generalized ansatz for the reduction of the fields transforming in a non-trivial representation of $\mathrm{G}$. The ansatz which was fist introduced by Scherk and Schwarz in [1] is

$$
\hat{\phi}\left(x^{\mu}, y^{m}\right)=g\left(y^{m}\right) \phi\left(x^{\mu}\right)
$$

where $\hat{\phi}$ is a generic field transforming under $\mathrm{G}$ as $\hat{\phi} \rightarrow g \hat{\phi}$ and $y^{m}, m=1, \cdots, d$ are coordinates on $T^{d}$ so that $g: T^{d} \rightarrow \mathrm{G}$. The ansatz (1.1) is equivalent to an expansion of the fields in terms of the harmonics of $T^{d}$ followed by a consistent truncation to the zero modes with a twisted boundary condition for $\hat{\phi}$ (as opposed to the periodic boundary conditions imposed by the standard Kaluza-Klein ansatz). As $\hat{\phi}$ traverses a cycle of $T^{d}$ parameterized by $0 \leq \tau_{i} \leq 2 \pi R_{i}, i=1, \cdots, d$ it picks up a monodromy $\Omega_{i}(g)$ so that the twisted boundary condition is

$$
\hat{\phi}\left(x^{\mu}, \tau_{i}=2 \pi R_{i}\right)=\Omega_{i} \hat{\phi}\left(x^{\mu}, \tau_{i}=0\right) .
$$

The monodromies introduce in lower dimensions a non-abelian gauge algebra, mass parameters and a scalar potential. The G-invariance of the higher dimensional theory ensures that the $y$-dependence cancels out in the lower dimensional action and the reduction is consistent in the sense that the solutions to the lower dimensional field equations can be 
uplifted to become solutions of the higher dimensional ones. In the recent literature, such dimensional reductions are usually called reductions with duality twists [2].

Later in [3], Scherk and Schwarz introduced a related scheme of generalized dimensional reduction, where now the global symmetry exploited is 'internal' as opposed to the 'external' symmetries of [1]. In the terminology of [1] and [3], the internal symmetries are the geometric symmetries associated with the internal manifold, while the external symmetries act on the spinor and p-form fields. The scheme introduced in [3] can be described as the dimensional reduction on a d-dimensional parallelizable manifold $\mathrm{X}$ with well-defined nowhere vanishing basis one-forms

$$
\eta^{m}=U_{n}^{m}(y) d y^{n},
$$

where $y^{m}$ are coordinates on $\mathrm{X}$ and $U_{n}^{m}(y)$ is a matrix element of the internal symmetry group G. The one-forms $\eta^{m}$ satisfy

$$
d \eta^{m}+\frac{1}{2} C_{n p}^{m} \eta^{n} \wedge \eta^{p}=0
$$

with coefficients

$$
C_{n p}^{m}=-2\left(U^{-1}\right)_{n}^{r}\left(U^{-1}\right)_{p}^{s} \partial_{[r} U_{s]}^{m} .
$$

Consistency requires $C_{n p}^{m}$ to be constant, which in turn implies that they are the structure constants of the Lie algebra of $\mathrm{G}$. Then locally the internal space $\mathrm{X}$ has the structure of the group manifold of $\mathrm{G}$. Globally $\mathrm{X}=\mathrm{G} / \Gamma$ where $\Gamma$ is a discrete subgroup of $\mathrm{G}$ [ [ब and hence its structure can be quite different from the group manifold. It is common in the literature to refer to such reductions as Scherk-Schwarz reductions and the internal space $\mathrm{X}$ as the twisted torus. In this paper, we are mainly interested in the local structure, so for our purposes here a twisted torus is a group manifold, where the group can be and in general is non-compact. Like with the reductions with duality twists, reductions on twisted tori too introduce in lower dimensions a non-abelian gauge algebra, mass parameters and a scalar potential.

As was already mentioned in [1] and [3], in some cases dimensional reduction on a twisted torus can be equivalent to a standard Kaluza-Klein reduction followed by a dimensional reduction with a duality twist. Indeed, after a Kaluza-Klein reduction, the internal symmetries associated with the geometry of the internal manifold are promoted to the external symmetries of the lower dimensional theory, which then can be exploited in a subsequent reduction with a duality twist. For example, consider a theory compactified on a two torus $T^{2}$. The lower dimensional theory has an $S L(2, \mathbb{R})$ symmetry as part of its global symmetry group, as $S L(2, \mathbb{R})$ is the large diffeomorphism group of $T^{2}$. In the spectrum of the theory there exists two scalar fields, $\tau_{1}$ and $\tau_{2}$, which correspond to the moduli parameterizing the shape of the internal $T^{2}$, transforming under $S L(2, \mathbb{R})$ through fractional linear transformations. Now, in a further compactification on a circle $S^{1}$ we can introduce a duality-twisted ansatz for these fields as in (1.1), where $g(y)$ is in $S L(2, \mathbb{R})$. From the point of view of the parent theory, this is nothing but a compactification on a three dimensional twisted torus with the metric

$$
d s^{2}=(2 \pi R)^{2} d y^{2}+\frac{A}{\tau_{2}(y)}\left|\tau(y) d x_{1}+d x_{2}\right|^{2},
$$


from which we can check that the basis 1 -forms $\eta^{m}$ satisfy $(1.4)^{1}$. Here $y$ parameterizes the $S^{1}, R$ is the radius of $S^{1}, A$ is the area of $T^{2}$ and $\tau=\tau_{1}+i \tau_{2}$. We will discuss this in more detail in section four.

The purpose of this paper is to consider twisted dimensional reductions on a group manifold $\mathrm{G}$, where the twist is in a duality group $\tilde{G}$ and examine the conditions for which the ansatz (1.1) yields a consistent dimensional reduction. We find that the Lie algebra (of $\tilde{G}$ ) valued one-form $A=\tilde{g}^{-1} d \tilde{g}=A_{m}(y) \eta^{m}$ (with $\tilde{g} \in \tilde{G}$ ) should have constant components $A_{n}(y)=M_{n}$ so that the $y$-dependence cancels out in the lower dimensional action and the field equations. As soon as we impose the condition that $M_{n}$ must be constant elements of the Lie algebra of $\tilde{G}$, not depending on the coordinates $y^{m}$ of $G$, we see that $M_{n}$ must also satisfy the following commutation relations

$$
\left[M_{n}, M_{p}\right]=C^{q}{ }_{n p} M_{q}
$$

This follows from the fact that the 1-form $A$, being of the form $A=\tilde{g}^{-1} d \tilde{g}$ satisfies the zero curvature condition

$$
d A+A \wedge A=0 .
$$

We also find, the condition (1.7) ensures the closure of the lower dimensional gauge algebra arising from the $\tilde{G}$-twisted reduction on $G$. At this point, an important question arises as to whether $A$ is pure gauge globally or only locally. If $\tilde{g}$ is single-valued on $G$ so that $A$ is pure gauge globally, then $A$ can be gauge transformed to a zero connection, rendering our dimensional reduction equivalent to a standard group manifold reduction on $G$, with no $\tilde{G}$ twist at all. However, if $G$ is not simply connected with $\pi_{1}(G) \neq 0$, then one can introduce non-trivial monodromies for the connection $A$ over the cycles of $G$, which then introduces twisted boundary conditions for the fields charged under the duality group $\tilde{G}$. In this case, $M_{n}$ introduces as usual the mass terms and the gauge parameters in the lower dimensional theory.

The plan of the paper is as follows. In the next section we briefly review the standard dimensional reduction on a group manifold. In section 3 we study the twisted dimensional reduction on a group manifold $\mathrm{G}$ of a particular $\tilde{G}$-invariant theory of gravity coupled to scalars and p-form fields. We find the consistency conditions for the cancellation of the $y$-dependence and the closure of the lower dimensional gauge algebra. In section 4 we turn to our main interest: unimodular, non-semi-simple group manifolds of dimension three. We review in this section that all such manifolds are locally isomorphic to a twisted torus with the metric (1.6), where $\tau(y)$ is given by (1.1) with $g(y)$ in a certain conjugacy class of SL(2). In section 5, we consider the low energy effective field theory of eight dimensional type II string theory. In this dimension, the U-duality group is $\mathrm{SL}(2) \times \mathrm{SL}(3)$. Dimensionally reducing on a three dimensional unimodular, non-semi-simple group manifold $\mathrm{G}$ with an SL(3) twist, we obtain in five dimensions a new gauged supergravity with mass terms and a scalar potential. We conclude with discussions in section 6 .

\footnotetext{
${ }^{1}$ Duality-twisted reductions are classified with respect to the conjugacy classes of the duality group. In each conjugacy class of $S L(2, \mathbb{R})$ a representative can be chosen such that $C_{n p}^{m}$ in $(1.4)$ are constants. See section 4 .
} 


\section{Dimensional Reduction on a Group Manifold}

The metric ansatz which leads to a consistent dimensional reduction from $D+d$ to $D$ dimensions on a $d$-dimensional group manifold $\mathrm{G}$ is the following

$$
\hat{d s}^{2}=e^{2 \alpha \phi} d s^{2}+e^{2 \beta \phi} \mathcal{M}_{m n}\left(\eta^{m}+\mathcal{A}_{\mu}^{m} d x^{\mu}\right)\left(\eta^{n}+\mathcal{A}_{\mu}^{n} d x^{\mu}\right)
$$

Here $y^{m}$ are the coordinates on the group manifold, which consists of the group elements $g\left(y^{m}\right)=g \in G$. That is, we have a group element of $G$ corresponding to each point on the group manifold. The $\eta^{m}(y)=\eta_{n}^{m}(y) d y^{n}$ with $\eta_{n}^{m}(y) \in G$ are the basis 1 -forms on $G, d s^{2}$ is the metric on the $D$ dimensional space-time, $\phi$ is the dilaton and the vectors $\mathcal{A}^{n}=\mathcal{A}_{\mu}^{n} d x^{\mu}$ are the $d$ graviphotons. $\mathcal{M}$ is a scalar matrix parameterizing the coset $S L(d, \mathbb{R}) / S O(d)$ corresponding to $d-1$ dilatons and $d(d-1) / 2$ axions. It is convenient to set the values of $\alpha$ and $\beta$ to

$$
\alpha^{2}=\frac{d}{2(D+d-2)(D-2)}, \quad \beta=-\frac{(D-2) \alpha}{d}
$$

so that the lower dimensional Einstein-Hilbert action has the conventional form. The internal part of (2.1) corresponds to the left invariant metric of a group manifold

$$
d s_{G}^{2}=e^{2 \beta \phi} \mathcal{M}_{m n} \eta^{m} \eta^{n} .
$$

In order for the lower dimensional theory to be independent of the internal coordinates, the internal dependence of $\eta^{m}(y)=\eta_{n}^{m}(y) d y^{n}$ should be chosen such that

$$
t_{m} \eta_{n}^{m} d y^{n}=g^{-1} d g
$$

for group elements $g=g\left(y^{m}\right)$, where $t_{m}$ forms a basis for the Lie algebra of $G$. As a result, $\eta^{m}$ are the left invariant Maurer-Cartan forms of a group manifold G, satisfying the condition (1.4) with constant structure constants $C_{n p}^{m}$. The metric ansatz (2.1) yields a consistent reduction of the $D+d$ dimensional Einstein-Hilbert action to $D$ dimensions, provided that the group $G$ is unimodular ${ }^{2}$. If $G$ is not unimodular, then there is a consistent reduction at level of field equations only [5, 6].

From the dimensional reduction of the $D+d$ dimensional Einstein-Hilbert action on a $d$ dimensional unimodular group $G$, one obtains the following Lagrangian in $D$ dimensions

$$
\mathcal{L}_{E H}=R * 1+\frac{1}{4} \operatorname{Tr}\left(D \mathcal{M} \wedge * D \mathcal{M}^{-1}\right)-\frac{1}{2} d \phi \wedge * d \phi-\frac{1}{4} e^{2(\alpha-\beta) \phi} \mathcal{F}^{m} \mathcal{M}_{m n} \wedge * \mathcal{F}^{n}-V
$$

Here

$$
\begin{gathered}
\mathcal{F}^{m}=d \mathcal{A}^{m}-\frac{1}{2} C^{m}{ }_{n p} \mathcal{A}^{n} \wedge \mathcal{A}^{p}, \\
D \mathcal{M}_{m n}=d \mathcal{M}_{m n}+2 C^{p}{ }_{q(m} \mathcal{A}^{q} \mathcal{M}_{n) p}
\end{gathered}
$$

\footnotetext{
${ }^{2}$ This means that the adjoint representation of the group has unit determinant. At the level of the Lie algebra, this implies that the structure constants are traceless, i.e, $C_{m n}^{m}=0$. Equivalently, any leftinvariant measure on $\mathrm{G}$ is also right-invariant, so all top-dimensional forms on $G$ are proportional up to a factor which does not depend on the coordinates on $G$. All compact groups and semi-simple groups (compact or not) are necessarily unimodular [5].
} 
and the scalar potential $V$ is

$$
V=\frac{1}{4} e^{-2(\alpha-\beta) \phi}\left[2 \mathcal{M}^{n q} C^{p}{ }_{m n} C_{p q}^{m}+\mathcal{M}^{m q} \mathcal{M}^{n r} \mathcal{M}_{p s} C^{p}{ }_{m n} C^{s}{ }_{q r}\right] .
$$

If the $D+d$ dimensional theory also includes p-form fields $B_{(p)}$, the corresponding ansatz for their reduction is

$$
\hat{B}_{(p)}=B_{(p)}+B_{(p-1) m} \wedge h^{m}+\cdots+\frac{1}{(p-k) !} B_{(k) m_{1} \cdots m_{p-k}} \wedge h^{m_{1}} \wedge \cdots \wedge h^{m_{p-k}} .
$$

Here $h^{m}=\eta^{m}+\mathcal{A}^{m}$ and $k$ is the larger of zero and $p-d$. The hatted fields on the left hand side are $D+d$ dimensional, whereas the unhatted fields $B_{(q)}$ on the right hand side are $D$ dimensional and do not depend on the $d$ internal coordinates for the consistency of the ansatz. For more details on group manifold reductions, see for example [5, 6].

\section{Twisted Reductions on a Group Manifold}

In the toroidal reduction of a $\tilde{G}$-invariant theory with a duality twist with an ansatz of the form (1.1), an important consistency criterium is that $\tilde{g}(y)^{-1} d \tilde{g}(y)$ should be a constant 1-form. This condition ensures that the dependence on the internal coordinates cancels out in the lower dimensional action and field equations. When the internal space is a circle an obvious consistent choice is $\tilde{g}(y)=e^{M y}$, with $M$ in the Lie algebra of $\tilde{G}$. For the generalization to a $d$ dimensional torus $T^{d}$ the appropriate choice is

$$
\tilde{g}\left(y^{1}, \cdots, y^{n}\right)=e^{M_{1} y^{1}+\cdots+M_{d} y^{d}}
$$

where $y^{m}$ are coordinates on $T^{d}$. The matrices $M_{n}$ in (3.1) are required to commute, so that the $y$ dependence cancels out and the gauge algebra closes in the lower dimensional theory. In this section we examine the case in which the internal space is a group manifold $G$. For this purpose, we study a particular type of Lagrangian for simplicity. Namely, we consider the group manifold reduction of a theory of gravity coupled to scalars in the coset $\tilde{G} / H$ (where $H$ is the maximally compact subgroup of $\tilde{G}$ ) and a set of $r n-1$ form gauge potentials $\hat{B}_{(p)}^{a}$ with $n$-form field strengths $\hat{H}_{(p+1)}^{a}=d \hat{B}_{(p)}^{a}, a=1, \ldots, r$, transforming in a real $r$-dimensional representation of the symmetry group $\tilde{G}$. This example will play a central role in the coming sections. The Lagrangian we will study is

$$
\mathcal{L}=R \hat{*} 1+\frac{1}{4} \operatorname{tr}\left(d \hat{\mathcal{K}} \wedge \hat{*} d \hat{\mathcal{K}}^{-1}\right)-\frac{1}{2} \hat{H}_{(p+1)}^{t} \hat{\mathcal{K}}^{-1} \wedge \hat{*} \hat{H}_{(p+1)}
$$

Here $\hat{\mathcal{K}}$ is an $r \times r$ matrix of scalar fields which act as a metric on the coset space $\tilde{G} / H$. The Lagrangian (3.2) is invariant under the rigid $\tilde{G}$ symmetry

$$
\hat{B}_{(p)} \rightarrow L \hat{B}_{(p)}, \quad \hat{\mathcal{K}} \rightarrow L \hat{\mathcal{K}} L^{t}
$$

where $L^{a}{ }_{b}$ is a $\tilde{G}$-transformation in the $\mathbf{r}$ representation, and the space-time metric is invariant. The invariance of the metric means that the ansatz for the dimensional reduction of the metric is the same as the standard ansatz for the group manifold reduction, which 
we presented in (2.1). On the other hand, the ansatz for the reduction of the scalar and the p-form fields are dictated by their transformation $(3.3)$ under $\tilde{G}$, so that we have

$$
\begin{gathered}
\hat{\mathcal{K}}(x, y)=\tilde{g}(y) \mathcal{K}(x) \tilde{g}^{t}(y), \\
\hat{B}_{(p)}(x, y)=\tilde{g}(y) \hat{B}_{(p)}(x),
\end{gathered}
$$

where $\hat{B}_{(p)}(x)$ on the right hand side of $(3.5)$ is as in (2.9) and $\tilde{g}(y) \in \tilde{G}$. Note that $y^{m}$ here are coordinates on $G$, now that our internal space is the group manifold $G$. In other words, $\tilde{g}$ is a map from the group manifold to the duality group $\tilde{G}: \tilde{g}: G \rightarrow \tilde{G}$. In the next subsection, we will see that imposing the condition for $\tilde{g}^{-1} d \tilde{g}$ to be a constant 1 -form is necessary and also sufficient in order for the dependence on the internal coordinates $y^{m}$ to cancel out in the $D$ dimensional Lagrangian. Then in the following subsection, we will see that a new condition has to be imposed for the lower dimensional gauge algebra to close.

\subsection{Action}

In this section we impose the condition that $\tilde{g}^{-1} d \tilde{g}$ has constant components with respect to the Maurer-Cartan forms of the group manifold $G$, so that it can be written as

$$
\tilde{g}^{-1} d \tilde{g}=M_{n} \eta^{n}
$$

where $M_{n}$ are constant elements of the Lie algebra of $\tilde{G}$.

The ansatz (3.5) for the reduction of the p-form fields $\hat{B}_{(p)}$ implies for the field strength $\hat{H}_{(p+1)}$

$$
\begin{aligned}
\hat{H}_{(p+1)}\left(x^{\mu}, y\right) & =d \hat{B}_{(p)}\left(x^{\mu}, y\right)=d \tilde{g}(y) \hat{B}_{(p)}\left(x^{\mu}\right)+\tilde{g}(y) d \hat{B}_{(p)}\left(x^{\mu}\right) \\
& =\tilde{g}(y)\left[d \hat{B}_{(p)}\left(x^{\mu}\right)+\tilde{g}^{-1} d \tilde{g}(y) \hat{B}_{(p)}\left(x^{\mu}\right)\right]
\end{aligned}
$$

When we insert (3.7) and (3.4) in the kinetic term for the p-form fields in (3.2), we see that it is independent of $y$ as the overall $\tilde{g}(y)$ factor cancels out due to the global $\tilde{G}$ invariance of the action, and $\tilde{g}^{-1} d \tilde{g}$ is independent of $y$ by (3.6). Now we check the kinetic term for the scalar fields

$$
\mathcal{L}_{s}=\frac{1}{4} \operatorname{tr}\left(d \hat{\mathcal{K}} \wedge \hat{*} d \hat{\mathcal{K}}^{-1}\right)
$$

From the Scherk-Schwarz ansatz (3.4) for the scalar fields we see

$$
d \hat{\mathcal{K}}=d \tilde{g} \mathcal{K} \tilde{g}^{t}+\tilde{g} d \mathcal{K} \tilde{g}^{t}+\tilde{g} \mathcal{K} d \tilde{g}^{t}=\tilde{g}\left(M_{p} \mathcal{K} \eta^{p}+d \mathcal{K}+\mathcal{K} M_{p}^{t} \eta^{p}\right) \tilde{g}^{t}
$$

Similarly,

$$
d \hat{\mathcal{K}}^{-1}=\left(\tilde{g}^{t}\right)^{-1}\left(-M_{p}^{t} \mathcal{K}^{-1} \eta^{p}+d \mathcal{K}^{-1}-\mathcal{K}^{-1} M_{p} \eta^{p}\right) \tilde{g}^{-1},
$$

where we have used $d\left(\tilde{g}^{-1}\right) \tilde{g}=-\tilde{g}^{-1} d \tilde{g}=-M_{n} \eta^{n}$. The overall $\tilde{g}$ factors cancel out in the action due to invariance of the Lagrangian under (3.3). As the mass matrices $M_{n}$ are constant, we conclude that the kinetic term for the scalar fields is also independent of the internal coordinates. 


\subsection{Gauge Algebra}

In this section we choose $p=2$ for simplicity. This is also the most interesting case for us, as we will see in section 5 . In the dimensional reduction of a $\tilde{G}$-invariant theory of the form (3.2), one obtains three sets of gauge fields in the reduced theory. These are the fields $B_{(2)}^{a}$ and $B_{(1) m}^{a}$ coming from the reduction of the 2 -form fields $B_{(2)}^{a}$ and the vector fields $\mathcal{A}^{m}$ coming from the reduction of the metric. Here $m$ runs from 1 to $d$, where $d$ is the dimension of the internal twisted torus. We denote the generators of the corresponding gauge transformations and the gauge parameters $\left\{Y^{a}, X^{m a}, Z_{m}\right\}$ and $\left\{\Lambda_{(1)}^{a}, \Lambda_{(0) m}^{a}, \omega^{m}\right\}$, respectively. For a reduction on a twisted torus, the gauge transformation for the vector fields are always of the form [3]

$$
\delta \mathcal{A}^{m}=d \omega^{m}+C_{n p}^{m} \omega^{n} \mathcal{A}^{p} .
$$

Note that this implies that the $h^{m}=\eta^{m}+\mathcal{A}^{m}$ always transform covariantly

$$
\delta h^{m}=C_{n p}^{m} \omega^{n} h^{p}
$$

as we have $i_{Z} \eta^{m}=-\omega^{m}$ and

$$
\delta \eta^{m}=d i_{Z} \eta^{m}+i_{Z} d \eta^{m}=-d \omega^{m}+C_{n p}^{m} \omega^{n} \eta^{p} .
$$

Before proceeding to compute the gauge transformations, let us first write down the field strengths for gauge fields in lower dimensions. From the ansatz for the 2-form field $B_{2}$

$$
\hat{B}_{(2)}\left(x^{\mu}, y^{n}\right)=\tilde{g}\left(y^{n}\right)\left[B_{(2)}\left(x^{\mu}\right)+B_{(1) m}\left(x^{\mu}\right) \wedge h^{m}+\frac{1}{2 !} B_{(0) m n}\left(x^{\mu}\right) h^{m} \wedge h^{n}\right],
$$

we find for the field strength $\hat{H}_{(3)}=d \hat{B}_{(2)}$

$$
\begin{aligned}
\hat{H}_{(3)}\left(x^{\mu}, y^{n}\right)= & \tilde{g}\left(y^{n}\right)\left[H_{(3)}\left(x^{\mu}\right)+H_{(2) n}\left(x^{\mu}\right) \wedge h^{n}\right. \\
& \left.+\frac{1}{2 !} H_{(1) n p}\left(x^{\mu}\right) \wedge h^{n} \wedge h^{p}+\frac{1}{3 !} H_{(0) m n p}\left(x^{\mu}\right) h^{m} \wedge h^{n} \wedge h^{p}\right],
\end{aligned}
$$

where

$$
\begin{aligned}
& H_{(3)}=d B_{(2)}-B_{(1) m} \wedge \mathcal{F}^{m}-M_{n} B_{(2)} \wedge \mathcal{A}^{n} \\
& H_{(2) n}=d B_{(1) n}+B_{(1) m} C_{n p}^{m} \mathcal{A}^{p}+B_{(0) m n} \wedge \mathcal{F}^{m}+M_{n} B_{(2)}+M_{p} B_{(1) n} \mathcal{A}^{p} \\
& H_{(1) n p}=d B_{(0) n p}+B_{(1) m} C_{n p}^{m}+B_{(0) m[n} C_{p] r}^{m} \mathcal{A}^{r}-M_{[n} B_{(1) p]}-M_{r} B_{(0) n p} \mathcal{A}^{r} \\
& H_{(0) n p r}=M_{[n} B_{(0) p r]}+C_{[n p}^{m} B_{(0) r] m}
\end{aligned}
$$

Here

$$
\mathcal{F}^{m}=d \mathcal{A}^{m}-\frac{1}{2} C^{m}{ }_{n p} \mathcal{A}^{n} \wedge \mathcal{A}^{p},
$$

and we have used

$$
d h^{m}=d \eta^{m}+d \mathcal{A}^{m}=\mathcal{F}^{m}+C^{m}{ }_{n p} \mathcal{A}^{n} \wedge h^{p}-\frac{1}{2} C^{m}{ }_{n p} h^{n} \wedge h^{p} .
$$


Note that $\mathcal{F}^{m}$ varies covariantly

$$
\delta \mathcal{F}^{m}=C_{n p}^{m} \omega^{n} \mathcal{F}^{p} .
$$

The terms in (3.16) involving the structure constants $C_{n p}^{m}$ are due to the twisted internal geometry, whereas the terms involving the mass matrices $M_{p}$ are due to the twisted ansatz for the 2-form field $B_{(2)}$.

Next we find the gauge transformations of the 2-form, 1-form and the scalar fields coming from the reduction of $\hat{B}_{(2)}$ inherited from its diffeomorphism invariance and the gauge transformation $\delta \hat{B}_{(2)}=d \hat{\Lambda}_{(1)}\left(x^{\mu}, y\right)$. Let us start with the latter. As we are using a generalized ansatz (3.14) for the reduction of $\hat{B}_{(2)}$, the ansatz for the higher dimensional gauge parameter $\hat{\Lambda}_{(1)}\left(x^{\mu}, y^{m}\right)$ should be

$$
\hat{\Lambda}_{(1)}\left(x^{\mu}, y^{m}\right)=\tilde{g}\left(y^{m}\right)\left[\Lambda_{(1)}\left(x^{\mu}\right)+\Lambda_{(0) m}\left(x^{\mu}\right) \wedge h^{m}\right] .
$$

From 3.20 ) we find

$$
\begin{aligned}
d \hat{\Lambda}_{(1)}= & \tilde{g}(y)\left[d \Lambda_{(1)}+\Lambda_{(0) m} \wedge \mathcal{F}^{m}+M_{p} \Lambda_{(1)} \mathcal{A}^{p}\right. \\
& +\left(d \Lambda_{(0) m}+\Lambda_{(0) n} C^{n}{ }_{p m} \mathcal{A}^{p}-M_{m} \Lambda_{(1)}-M_{p} \Lambda_{(0) m} \mathcal{A}^{p}\right) \wedge h^{m} \\
& \left.+\frac{1}{2 !}\left(-\Lambda_{(0) p} C^{p}{ }_{m n}+M_{[m} \Lambda_{(0) n]}\right) h^{m} \wedge h^{n}\right]
\end{aligned}
$$

Comparing this to $\delta \hat{B}_{(2)}$ with the ansatz (3.14) we find

$$
\begin{aligned}
& \delta B_{(2)}=d \Lambda_{(1)}+M_{p} \Lambda_{(1)} \mathcal{A}^{p}+\Lambda_{(0) m} \mathcal{F}^{m} \\
& \delta B_{(1) m}=d \Lambda_{(0) m}-\Lambda_{(0) n} C^{n}{ }_{m p} \mathcal{A}^{p}-M_{m} \Lambda_{(1)}-M_{p} \Lambda_{(0) m} \mathcal{A}^{p} \\
& \delta B_{(0) m n}=-\Lambda_{(0) p} C^{p}{ }_{m n}+M_{[m} \Lambda_{(0) n]}
\end{aligned}
$$

Now we find the gauge transformations inherited from the diffeomorphism invariance of $\hat{B}_{(2)}$.

$$
\begin{aligned}
\delta_{Z} \hat{B}_{(2)}= & \mathcal{L}_{Z} \hat{B}_{(2)}=0=\mathcal{L}_{Z}\left[\tilde{g}(y)\left(B_{(2)}+B_{(1) m} \wedge h^{m}+\frac{1}{2 !} B_{(0) m n} h^{m} \wedge h^{n}\right)\right] \\
= & \left(\mathcal{L}_{Z} \tilde{g}(y)\right)\left(B_{(2)}+B_{(1) m} \wedge h^{m}+\frac{1}{2 !} B_{(0) m n} h^{m} \wedge h^{n}\right) \\
& +\tilde{g}(y) \mathcal{L}_{Z}\left(B_{(2)}+B_{(1) m} \wedge h^{m}+\frac{1}{2 !} B_{(0) m n} h^{m} \wedge h^{n}\right) .
\end{aligned}
$$

Noting

$$
\mathcal{L}_{Z} \tilde{g}(y)=i_{Z} d \tilde{g}(y)+d i_{Z} \tilde{g}(y)=i_{Z} d \tilde{g}(y)=\tilde{g} i_{Z} \tilde{g}^{-1} d \tilde{g}(y)=-\tilde{g} M_{p} \omega^{p},
$$

and taking into account (3.12) we obtain

$$
\begin{aligned}
& \delta_{Z} B_{(2)}=M_{p} \omega^{p} B_{(2)} \\
& \delta_{Z} B_{(1) m}=M_{p} \omega^{p} B_{(1) m}-B_{(1) p} C^{p}{ }_{n m} \omega^{n} \\
& \delta_{Z} B_{(0) m n}=M_{p} \omega^{p} B_{(0) m n}+B_{(0) p[n} C^{p}{ }_{m] q} \omega^{q}
\end{aligned}
$$


One can check that the field strengths (3.16) are indeed invariant under the gauge transformations (3.22) and transform covariantly under (3.24).

Now we have to check that the gauge algebra closes. At this point, we find that we need to impose a new condition in addition to the constancy of the mass matrices $M_{n}$. Namely, $M_{n}$ has to satisfy the following commutation relations

$$
\left[M_{n}, M_{p}\right]=C^{q}{ }_{n p} M_{q}
$$

This means that $M_{n}$, which are elements of the Lie algebra of $\tilde{G}$ should form a representation of the Lie algebra of $G$. Obviously, this is possible only if $G$ is a subgroup of $\tilde{G}$. Note that we should have anticipated the condition (3.25) already in the previous subsection, when we imposed that the mass matrices $M_{n}$ in (3.6) do not depend on the coordinates $y^{m}$ of $G$. To see why, we should first note that the 1 -form $A \equiv \tilde{g}^{-1} d \tilde{g}$ automatically satisfies the zero curvature condition

$$
d A+A \wedge A=0 .
$$

On the other hand, the basis 1-forms $\eta^{m}$ satisfy the Maurer-Cartan equation (1.4). These two equations are compatible with constant $M_{p}$ if and only if the commutation relation (3.25) is satisfied. Therefore, our analysis of the gauge algebra shows that it is consistent to require $A$ to be a constant, flat $\tilde{G}$-connection on $G$. At this point, we see that the topology of $G$ plays an important role in analyzing as to whether it is possible to introduce non-trivial duality twists on the group manifold $G$. More precisely, we see that if $\pi_{1}(G)=0$ so that the group manifold is simply-connected, then the flat connection $A$ is pure gauge globally, giving a dimensional reduction equivalent to a standard group manifold reduction with no duality twist. This follows from the fact that the moduli space of flat $\tilde{G}$-connections on $G$ (modulo smooth gauge transformations) can be identified with $\operatorname{Hom}\left(\pi_{1}(G), \tilde{G}\right) / \tilde{G}$, where $\tilde{G}$ acts by conjugation. Therefore, it is crucial that $G$ is non-simply-connected so that the non-trivial Wilson lines/holonomies of the connection $A$ over the cycles of $G$ introduce twisted boundary conditions for the fields charged under $\tilde{G}$ (with analogy to a twisted reduction on $T^{d}$ in which case we have the twisted boundary conditions (1.2)). So far our analysis has been only local and we will not be studying the global issues in the rest of the paper, either. However, we will have a bit more to say on the non-simply-connectedness condition on the internal space, when we restrict ourselves to particular examples in the next section.

When the condition 3.25 is satisfied, the gauge algebra has the following form ${ }^{3}$

$$
\begin{gathered}
{\left[Z_{n}, X^{m}\right]=C_{n q}^{m} X^{q}-M_{n} X^{m}} \\
{\left[Z_{p}, Y^{a}\right]=-M_{p b}^{a} Y^{b}} \\
{\left[Z_{p}, Z_{q}\right]=-C_{p q}^{r} Z_{r} .}
\end{gathered}
$$

Note that this algebra is different from the one that is obtained from the reduction on a twisted torus with constant flux for the 2 -form field $B_{(2)}^{a}$. In this case, it was shown in [1, 4]

\footnotetext{
${ }^{3}$ In finding the gauge algebra, we also have to use the condition $C_{[n p}^{m} C_{r] m}^{q}=0$, which follows immediately from the integrability of (1.4).
} 
that the gauge transformations coming from the higher dimensional gauge invariance of the 2-form field is still of the form (3.22), where now all the terms with the mass matrices $M_{p}$ are zero. On the other hand the gauge transformations coming from the diffeomorphism invariance of $B_{(2)}$ is of the form

$$
\begin{aligned}
& \delta_{Z} B_{(2)}=\frac{1}{2} K_{m n p} \omega^{p} \mathcal{A}^{m} \wedge \mathcal{A}^{n} \\
& \delta_{Z} B_{(1) m}=K_{m n p} \omega^{p} \mathcal{A}^{n}-B_{(1) p} C^{p}{ }_{n m} \omega^{n} \\
& \delta_{Z} B_{(0) m n}=K_{m n p} \omega^{p}+B_{(0) p[n} C^{p}{ }_{m] q} \omega^{q},
\end{aligned}
$$

where

$$
\frac{1}{3 !} K_{m n p} \eta^{m} \wedge \eta^{n} \wedge \eta^{p}
$$

with constant $K_{m n p}$ being the 3 -form flux introduced for the 2 -form field $B_{(2)}$. We see that (3.30) is equivalent to (3.24) only for special values of the gauge fields, namely when

$$
\begin{aligned}
& M_{p} B_{(2)}=\frac{1}{2} K_{p m n} \mathcal{A}^{m} \wedge \mathcal{A}^{n} \\
& M_{[p} B_{(1) m]}=-K_{p m n} \mathcal{A}^{n} \\
& M_{[p} B_{(0) m n]}=-K_{p m n}
\end{aligned}
$$

As a final remark, note that a gauge algebra of the form (3.27,3.28, 3.29) was obtained in [8], albeit in a completely different context. There they consider the compactifications of the heterotic string, the generators $Y^{a}$ are associated with the gauge invariance of the 16 vector fields in the Yang-Mills sector that already exist in 10 dimensions and $M_{p}$ correspond to the internal fluxes in the Yang-Mills sector.

\section{Three Dimensional Group Manifolds and Twisted Tori}

In this section we restrict ourselves to three dimensional group manifolds. The classification of three dimensional algebras was made long ago by Bianchi. As reviewed by [6] there are eleven inequivalent three dimensional algebras, two of which are one-parameter families. Among them, five are of Type B, meaning that they are not unimodular algebras. The six unimodular algebras of Type A include the two semi-simple algebras $s o(2,1)$ and $s o(3)$. We are interested here in the three non-abelian unimodular non-semisimple algebras: heis 3 , $i s o(1,1)$ and $i s o(2)$. The fourth unimodular, non-semi-simple algebra, which is the only abelian three dimensional algebra, $u(1)^{3}$ has the group manifold $T^{3}$ (after some discrete identifications, which we are not interested here as we study only the local structure), so the corresponding group manifold reduction is a standard Kaluza-Klein reduction on a three dimensional torus. As we mentioned before, we require unimodularity so that there is a consistent dimensional reduction at the level of the action. On the other hand, nonsemi-simplicity is required because then the corresponding group manifold has the local structure of a $T^{2}$ fibration over $S^{1}$ [9], [ [ [ , making it easier to analyze the twisted torus geometry. We will now study this structure, following closely the discussion in 4 . 
Consider a reduction on $T^{2}$ with metric

$$
d s_{2}^{2}=\frac{A}{\tau_{2}}\left|\tau d x_{1}+d x_{2}\right|^{2}
$$

where $\tau=\tau_{1}+i \tau_{2}$ is the complex structure modulus and $A$ is the area of $T^{2}$. The metric (4.1) can also be written as

$$
d s_{2}^{2}=H(\tau)_{a b} d x^{a} d x^{b}=d x^{t} H(\tau) d x,
$$

with

$$
H(\tau)=\frac{A}{\tau_{2}}\left(\begin{array}{cc}
|\tau|^{2} & \tau_{1} \\
\tau_{1} & 1
\end{array}\right) .
$$

The symmetry group associated with the large diffeomorphisms of $T^{2}$ is $G L(2, \mathbb{R})$, with the volume preserving subgroup $S L(2, \mathbb{R})$. The action of $S L(2, \mathbb{R})$ on the metric is

$$
H \rightarrow L H L^{t}, \quad x \rightarrow\left(L^{t}\right)^{-1} x
$$

with $L \in S L(2, \mathbb{R})$. This defines the transformation of the moduli through

$$
H\left(\tau^{\prime}\right)=L H(\tau) L^{t}
$$

If

$$
L=\left(\begin{array}{ll}
a & b \\
c & d
\end{array}\right) \in S L(2, \mathbb{R})
$$

then (4.5) is equivalent to

$$
\begin{aligned}
& \tau_{1} \longrightarrow \frac{a c\left(\tau_{1}^{2}+\tau_{2}^{2}\right)+(a d+b c) \tau_{1}+b d}{c^{2}\left(\tau_{1}^{2}+\tau_{2}^{2}\right)+2 d c \tau_{1}+d^{2}} \\
& \tau_{2} \longrightarrow \frac{\tau_{2}}{c^{2}\left(\tau_{1}^{2}+\tau_{2}^{2}\right)+2 d c \tau_{1}+d^{2}}
\end{aligned}
$$

The transformation (4.6) of the moduli can be written in the compact form

$$
\tau \rightarrow \frac{a \tau+b}{c \tau+d} \equiv L[\tau]
$$

which of course is nothing but the fractional linear transformation of the complex structure modulus of $T^{2}$ under the action of $S L(2, \mathbb{R})$.

After the dimensional reduction on $T^{2}$ the geometric internal symmetry $S L(2, \mathbb{R})$ of $T^{2}$ is promoted to become an external symmetry of the lower dimensional theory, which we can use to perform a further reduction on a circle with duality twist. One can introduce a twisted ansatz for the two massless scalar fields corresponding to the moduli $\tau_{i}$ through

$$
H(\tau(y))=s(y) H\left(\tau_{0}\right) s^{t}(y)
$$

with $s(y)$ a $y$ dependent $S L(2, \mathbb{R})$ element and $\tau_{0}$ is a constant value of the modulus. Here $y$ parameterizes the circle $S^{1}$. The ansatz (4.8) follows directly from the transformation (4.5) of $\tau$. Then the metric of the three dimensional total space is

$$
d s_{3}^{2}=d y^{2}+d x^{t} H(\tau(y)) d x=\left(\eta^{y}\right)^{2}+\eta^{t} H\left(\tau_{0}\right) \eta,
$$


where

$$
\eta^{y}=d y, \quad \eta^{a}(y)=\left(s^{t}(y)\right)^{a}{ }_{b} d x^{b},
$$

with $a, b=1,2$. The metric (4.9) is equivalent to (1.6).

The group structure of this space was studied in «4. The globally well-defined 1-forms (4.10) satisfy

$$
d \eta^{a}+\left(N^{t}\right)_{b}^{a} \eta^{y} \wedge \eta^{b}=0
$$

where $N$ is the Lie algebra element $s(y)=e^{N y}$. Then locally, the space with the metric (4.9) has the structure of a group manifold with Maurer-Cartan 1-forms (4.10). The associated Lie algebra is

$$
\left[t_{a}, t_{y}\right]=N_{a}^{b} t_{b}, \quad\left[t_{a}, t_{b}\right]=0 .
$$

It is well known that reductions with duality twists are classified according to the conjugacy classes of the duality group. The symmetry group $S L(2, \mathbb{R})$ of our interest here has three conjugacy classes: parabolic, elliptic and hyperpolic.

\section{Parabolic Conjugacy Class:}

In this case the group element $s(y)$ and the corresponding matrix $N$ are

$$
s(y)=\left(\begin{array}{cc}
1 & m y \\
0 & 1
\end{array}\right), \quad N=\left(\begin{array}{cc}
0 & m \\
0 & 0
\end{array}\right) .
$$

Then the Lie algebra (4.12) is the Heisenberg algebra heis 3 , also called the Bianchi II in the Bianchi classification scheme, which is a non-semi-simple algebra of Type A. The Maurer-Cartan 1-forms (4.10) are

$$
\eta^{1}=d x^{1}, \quad \eta^{2}=d x^{2}+m y d x^{1}, \quad \eta^{3}=d y .
$$

In summary, reducing on $T^{2}$ followed by a circle reduction with a duality twist in the parabolic conjugacy class of $S L(2, \mathbb{R})$ is locally equivalent to reducing from $D+3$ to $D$ dimensions on the group manifold of the Heisenberg algebra (also known as the nilmanifold) with the Maurer-Cartan 1-forms (4.14). Now suppose that the $D+3$ dimensional theory that we start with has a duality symmetry $\tilde{G}$ which contains the Heisenberg group as a subgroup. Then, as we saw in the previous section, it is possible to introduce a twisted ansatz for the dimensional reduction of the $D+3$ fields on the nilmanifold through a group element $\tilde{g}\left(x^{1}, x^{2}, y\right)$ provided that it satisfies (3.6). For consistency, the mass matrices $M_{n}$ must be constant and form a representation of the Heisenberg algebra. If $\tilde{G}$ is the Heisenberg group itself, then a convenient choice of $\tilde{g}$ is

$$
\tilde{g}=\left(\begin{array}{ccc}
1 & -q y & p x^{2} \\
0 & 1 & r x^{1} \\
0 & 0 & 1
\end{array}\right)
$$

Then the mass matrices are

$$
M_{1}=\left(\begin{array}{ccc}
0 & 0 & 0 \\
0 & 0 & r \\
0 & 0 & 0
\end{array}\right), \quad M_{2}=\left(\begin{array}{lll}
0 & 0 & p \\
0 & 0 & 0 \\
0 & 0 & 0
\end{array}\right), \quad M_{3}=\left(\begin{array}{ccc}
0 & -q & 0 \\
0 & 0 & 0 \\
0 & 0 & 0
\end{array}\right) .
$$


Here $p, q, r$ have dimension of mass and satisfy $\frac{q r}{p}=m$. One can check that $M_{n}$ form a representation of the Heisenberg algebra and that $\tilde{g}$ in (4.15) satisfies (3.6) with $M_{n}$ in (4.16) and $\eta^{m}$ in (4.14).

Elliptic Conjugacy Class:

In this case, the group element $s(y)$ and the corresponding matrix $N$ are

$$
s(y)=\left(\begin{array}{cc}
\cos m y & \sin m y \\
-\sin m y & \cos m y
\end{array}\right), \quad N=\left(\begin{array}{cc}
0 & m \\
-m & 0
\end{array}\right) .
$$

The basis 1-forms (4.10) are

$$
\eta^{1}=\cos m y d x^{1}-\sin m y d x^{2}, \quad \eta^{2}=\sin m y d x^{1}+\cos m y d x^{2}, \quad \eta^{3}=d y .
$$

The corresponding algebra (4.12) is that of $i s o(2)$ (Bianchi $\mathrm{VII}_{0}$ ), so we see that a $T^{2}$ reduction followed by a reduction on a circle with an $S L(2, \mathbb{R})$ twist in the elliptic conjugacy class is locally equivalent to group manifold reduction on $I S O(2)$. If we start with a $D+3$ dimensional theory invariant under a symmetry group $\tilde{G}$ that contains $I S O(2)$, then we can introduce a duality twist for the reduction of the $D+3$-dimensional fields through a group element $\tilde{g} \in \tilde{G}$ provided that $\tilde{g}$ satisfies (3.6) with constant $M_{n}$ forming a representation of $i s o(2)$. If $\tilde{G}$ is $I S O(2)$ itself, a convenient choice of $\tilde{g}$ is

$$
\tilde{g}=\left(\begin{array}{ccc}
\cos m y & \sin m y & p x^{1} \\
-\sin m y & \cos m y & p x^{2} \\
0 & 0 & 1
\end{array}\right)
$$

Then the mass matrices are

$$
M_{1}=\left(\begin{array}{lll}
0 & 0 & p \\
0 & 0 & 0 \\
0 & 0 & 0
\end{array}\right), \quad M_{2}=\left(\begin{array}{lll}
0 & 0 & 0 \\
0 & 0 & p \\
0 & 0 & 0
\end{array}\right), \quad M_{3}=\left(\begin{array}{ccc}
0 & m & 0 \\
-m & 0 & 0 \\
0 & 0 & 0
\end{array}\right) .
$$

Hyperbolic Conjugacy Class:

In this case, the group element $s(y)$ and the corresponding matrix $N$ are

$$
s(y)=\left(\begin{array}{cc}
e^{m y} & 0 \\
0 & e^{-m y}
\end{array}\right), \quad N=\left(\begin{array}{cc}
m & 0 \\
0 & -m
\end{array}\right) .
$$

The basis 1-forms (4.10) are

$$
\eta^{1}=e^{m y} d x^{1} \quad \eta^{2}=e^{-m y} d x^{2}, \quad \eta^{3}=d y .
$$

The corresponding algebra (4.12) is that of $i s o(1,1)$ (Bianchi $\left.\mathrm{VI}_{0}\right)$, so we see that a $T^{2}$ reduction followed by a reduction on a circle with an $S L(2, \mathbb{R})$ twist in the hyperbolic conjugacy class is locally equivalent to group manifold reduction on $I S O(1,1)$. If the $D+3$ dimensional theory we start with is invariant under a symmetry group $\tilde{G}$ that contains 
$I S O(1,1)$, then we can introduce a duality twist for the reduction of the $D+3$-dimensional fields through a group element $\tilde{g} \in \tilde{G}$ provided that $\tilde{g}$ satisfies (3.6) with constant $M_{n}$ forming a representation of $i s o(1,1)$. If $\tilde{G}$ is $I S O(1,1)$ itself, a convenient choice of $\tilde{g}$ is

$$
\tilde{g}=\left(\begin{array}{ccc}
e^{-m y} & 0 & p x^{1} \\
0 & e^{m y} & q x^{2} \\
0 & 0 & 1
\end{array}\right)
$$

Then the mass matrices are

$$
M_{1}=\left(\begin{array}{lll}
0 & 0 & p \\
0 & 0 & 0 \\
0 & 0 & 0
\end{array}\right), \quad M_{2}=\left(\begin{array}{lll}
0 & 0 & 0 \\
0 & 0 & q \\
0 & 0 & 0
\end{array}\right), \quad M_{3}=\left(\begin{array}{ccc}
-m & 0 & 0 \\
0 & m & 0 \\
0 & 0 & 0
\end{array}\right) .
$$

Note that we can introduce three independent mass parameters in this case, whereas it was only possible to introduce two independent mass parameters in the previous cases.

Before we close this section, we would like to make a remark on the global issues, or more precisely on the condition of non-simply-connectedness, which comes about from requiring that the connection $A=\tilde{g}^{-1} d \tilde{g}$ is not pure gauge globally. The requirement that the internal space has a non-trivial fundamental group is clearly a topological condition, so it cannot be explored by the local analysis we have pursued here. For example, corresponding to the Bianchi IX algebra so(3), there are two groups: $S O(3)$ and $S U(2)$. The latter is a simply-connected group with trivial fundamental group, whereas $S O(3)$ has $\pi_{1}(S O(3))=Z_{2}$. A more detailed analysis than we have carried out here, including the global issues was given in 体. Note that all three group manifolds that we have studied in this section are non-compact. Performing a dimensional reduction on a non-compact group manifold $G$ leads to a continuous spectrum in the lower dimensional theory. However, it is possible to consistently truncate this spectrum to a finite number of fields, yielding gauged supergravities if the higher dimensional theory that we start with is itself a supergravity theory. This can be extended to a compactification of string theory only if one can construct a compact internal space $X=G / \Gamma$ by compactifying $G$ by dividing out by the action of a discrete symmetry group $\Gamma \subset G$ [ [⿴囗. and $\mathrm{VI}_{a}$ it is possible to construct compact manifolds in this way 6$]^{4}$. Therefore, even if the non-compact group manifold that we start with is simply-connected so that $\pi_{1}(G)$ is trivial, the compact manifold $X=G / \Gamma$ that we construct from $G$ will have a non-trivial fundamental group $\pi_{1}(X) \approx \Gamma$. This then makes it possible to introduce non-trivial duality twists on $X$, as we discussed in section 3 .

\section{Example}

In this section we will study the dimensional reduction of a particular eight dimensional theory with a duality twist on a three dimensional unimodular, non-semi-simple group

\footnotetext{
${ }^{4}$ See [- 1 for the explicit forms of the discrete subgroups $\Gamma$ which one can use to compactify the three non-abelian, unimodular, non-semi-simple group manifolds we have considered here.
} 
manifold. As we saw in the previous section, there are three such group manifolds (other than the abelian $T^{3}$ ) and locally each has the structure of a twisted torus. The eight dimensional theory that we are interested in is the Type II supergravity, which has the duality symmetry $S L(2, \mathbb{R}) \times S L(3, \mathbb{R})[10$. This theory describes the low energy effective theory of Type IIA/IIB string theory compactified on $T^{2}$, or equivalently M-theory compactified on $T^{3}$ [11, 12]. It has a consistent truncation to the sector of $S L(2, \mathbb{R})$ singlets, which is described by the Lagrangian ${ }^{5}$

$$
\mathcal{L}_{8}=R * 1+\frac{1}{4} \operatorname{Tr}\left(d \mathcal{K} \wedge * d \mathcal{K}^{-1}\right)+\frac{1}{2} H_{(3)}^{t} \mathcal{K}^{-1} \wedge * H_{(3)}+\frac{1}{6} \epsilon^{a b c} H_{(3) a} \wedge H_{(3) b} \wedge B_{(2) c}
$$

where $a, b, c=1,2,3$ and $H_{(3)}=d B_{(2)}$ is the 3 -vector

$$
H_{(3)}=\left(\begin{array}{l}
d B_{(2) 1} \\
d B_{(2) 2} \\
d B_{(2) 3}
\end{array}\right) .
$$

From the M-theory point of view, the three 2-form fields $B_{(2) a}$ come from the reduction of the eleven dimensional 3-form field. The IIB origin of $B_{(2) a}$ is the two (one in the NS-NS sector and one in the R-R sector) 2-form fields that already exist in the massless spectrum of the ten dimensional IIB string theory and the self-dual 4-form field $C_{(4)}$. The dimensional reduction of $C_{(4)}$ yields in eight dimensions one 2-form field and one 4-form field, which can be dualized to a second 2-form field. Imposing the self-duality constraint reduces the number of 2 -form fields to one. The scalar matrix $\mathcal{K}$ in (5.1) represents the coset space $S L(3, \mathbb{R}) / S O(3)$ parameterized by the scalar fields coming from the reduction of the eleven dimensional metric. From the IIB point of view, two of the five scalars parameterizing $\mathcal{K}$ are the axion and the dilaton that exist in ten dimensions; two come from the reduction of the two ten dimensional 2-form fields and the fifth is the massless scalar associated with the volume modulus of $T^{2}$ (the scalar coming from the reduction of the 2-form field in the NS-NS sector combines with this fifth scalar to form the Kähler modulus of $T^{2}$ ). The Lagrangian (5.1) is manifestly invariant under the duality group $S L(3, \mathbb{R})$ under which $B_{(2)}$ and $\mathcal{K}$ transform as

$$
B_{(2)} \rightarrow \Gamma B_{(2)}, \quad \mathcal{K} \rightarrow \Gamma \mathcal{K} \Gamma^{t}, \quad \Gamma \in S L(3, \mathbb{R}) .
$$

One can use this $S L(3, \mathbb{R})$ symmetry to introduce a twisted ansatz for the dimensional reduction of the fields $B_{(2)}$ and $\mathcal{K}$ through

$$
\begin{gathered}
B_{(2)}\left(x^{\mu}, y^{m}\right)=\tilde{g}\left(y^{m}\right) B_{(2)}\left(x^{\mu}\right) \\
\mathcal{K}\left(x^{\mu}, y^{m}\right)=\tilde{g}\left(y^{m}\right) \mathcal{K}\left(x^{\mu}\right) \tilde{g}^{t}\left(y^{m}\right) .
\end{gathered}
$$

Here $y^{m}$ are the coordinates of the internal space and $\tilde{g}\left(y^{m}\right) \in \tilde{G}=S L(3, \mathbb{R})$. As we mentioned above, we would like to choose the internal space as a three dimensional unimodular,

\footnotetext{
${ }^{5}$ Here we are omitting the hats on the higher dimensional fields, hoping that the eight-dimensional fields here will not be confused with the five-dimensional fields in (5.6,5.9) and (5.10).
} 
non-semi-simple group manifold. We have seen in section 3 that this group manifold reduction with the twisted ansatz (5.3,5.4) is consistent only if the $s l(3, \mathbb{R})$-valued 1 -form $A \equiv \tilde{g}^{-1} d \tilde{g}$ is a constant 1 -form $A=M_{p} \eta^{p}$ with the mass matrices $M_{p}$ satisfying the commutation relations (3.25). One can easily find such $\tilde{g} \in S L(3, \mathbb{R})$. In fact, the group elements we introduced in (4.15), (4.19) and (4.23) are all elements of the group $S L(3, \mathbb{R})$. Therefore, it is possible to introduce non-trivial $S L(3, \mathbb{R})$ twists through these choices of $\tilde{g}$ for the dimensional reduction on the corresponding group manifolds. This gives a gauged supergravity theory in five dimensions, with the mass matrices determining the gauge and mass parameters and the scalar potential given by (4.16),(4.20) and (4.24) for the nilmanifold, $I S O(2)$ and $I S O(1,1)$ reductions respectively. Below we present the Lagrangian describing the resulting five dimensional gauged supergravity theory, leaving the details of the reduction to Appendix A.

The five dimensional Lagrangian is

$$
\mathcal{L}_{5}=\mathcal{L}_{E H}+\mathcal{L}_{S}+\mathcal{L}_{K}+\mathcal{L}_{C S}
$$

Here $\mathcal{L}_{E H}$ comes from the reduction of the Einstein-Hilbert term in (5.1) and is exactly of the form (2.5) as we are reducing on a group manifold. The eight dimensional metric is invariant under the duality symmetry $S L(3, \mathbb{R})$, so there is no correction to (2.5) due to the $S L(3, \mathbb{R})$ twist. The $\mathcal{L}_{S}$ term in (5.5) comes from the reduction of the scalars parameterizing $\mathcal{K}$ and is of the form

$$
\mathcal{L}_{S}=\frac{1}{4} \operatorname{Tr}\left(D \mathcal{K} \wedge * D \mathcal{K}^{-1}\right)-e^{4 \alpha \phi} V
$$

where

$$
\begin{aligned}
D \mathcal{K} & =d \mathcal{K}-M_{m} \mathcal{K} \mathcal{A}^{m}-\mathcal{K} M_{m}^{t} \mathcal{A}^{m} \\
D \mathcal{K}^{-1} & =d \mathcal{K}^{-1}+M_{m}^{t} \mathcal{K}^{-1} \mathcal{A}^{m}+\mathcal{K}^{-1} M_{m} \mathcal{A}^{m}
\end{aligned}
$$

and

$$
V=\frac{1}{2} \sum_{i} \operatorname{Tr}\left(M_{i}^{2}+\mathcal{K} M_{i}^{t} \mathcal{K}^{-1} M_{i}\right)
$$

Here $M_{i}=L_{i}{ }^{m} M_{m}$ with $L_{i}{ }^{m} L_{j}{ }^{n} \delta^{i j}=\mathcal{M}^{m n}$. The $m, n$ are curved indices for the internal space, whereas $i, j$ are the flat indices of the tangent space of the internal space. (See Appendix A for details.) $M_{m}$ are the mass matrices in (4.16), (4.20) or (4.24) depending on which unimodular, non-semisimple group manifold we pick to reduce on and $\mathcal{M}$ is the matrix in the coset space $S L(3, \mathbb{R}) / S O(3)$ parameterized by the five scalars coming from the reduction of the eight dimensional metric on the group manifold.

The dimensional reduction of the kinetic term for the fields $B_{(2)}$ in (5.1) yields in five dimensions the following kinetic terms

$$
\begin{aligned}
\mathcal{L}_{K}= & \frac{1}{2}\left(e^{-4 \alpha \phi} H_{(3) a} \mathcal{K}^{a b} \wedge * H_{(3) b}+H_{(2) a m} \mathcal{K}^{a b} \mathcal{M}^{m n} \wedge * H_{(2) b n}\right. \\
& \left.+e^{2 \alpha \phi} H_{(1) a}^{m} \mathcal{K}^{a b} \mathcal{M}_{m n} \wedge * H_{(1) b}^{n}+e^{8 \alpha \phi} H_{(0) a} \mathcal{K}^{a b} \wedge * H_{(0) b}\right) .
\end{aligned}
$$


Here $H_{(1)}^{m}=\epsilon^{m n p} H_{(1) n p}, H_{(0)}=\epsilon^{m n p} H_{(0) m n p}$ and $H_{(3)}, H_{(2) m}, H_{(1) n p}, H_{(0) m n p}$ are as in (3.16). Finally, the dimensional reduction of the topological term in (5.1) gives the $\mathcal{L}_{C S}$ term in 5.5$)$

$$
\mathcal{L}_{C S}=\epsilon^{a b c} \epsilon^{m n p}\left(H_{(2) a m} \wedge H_{(2) b n} \wedge B_{(1) c p}+H_{(3) a} \wedge H_{(1) b m n} \wedge B_{(1) c p}\right) .
$$

Note that there are two potential terms in (5.5). The first is for the scalar fields coming from the reduction of the eight dimensional metric, associated with the moduli parameterizing the internal space and is of the form (2.8). The second term (5.8) is for these geometric moduli and for the scalars that already exist in eight dimensions, parameterizing the matrix $\mathcal{K}$. As we mentioned above, from the point of view of M-theory, these are geometric moduli associated with the internal $T^{3}$ on which M-theory has been compactified to obtain the eight dimensional theory (5.1). On the other hand, from the point of view of IIB theory, this is a potential term for the axion-dilaton field, the Kähler modulus of the internal $T^{2}$ (on which IIB has been compactified) and the scalar field coming from the reduction of ten dimensional R-R 2-form field. The gauge algebra of our new five dimensional gauged supergravity theory $(5.5)$ is exactly the algebra that we presented in (3.27,3.28, 3.29).

\section{Outlook}

In this paper we have studied the consistency conditions for the dimensional reduction of a $\tilde{G}$-invariant theory with a duality twist in $\tilde{G}$ on a group manifold $G$. We have seen that if the duality twist is introduced through a group element $\tilde{g} \in \tilde{G}$, then the $\tilde{G}$-connection $A=\tilde{g}^{-1} d \tilde{g}$ on $G$ must be constant. So, if $\eta^{m}$ are basis 1-forms for the group manifold $G$ satisfying (1.4) then $A$ can be written as $A=M_{n} \eta^{n}$ with constant $M_{n}$ satisfying (3.25). $M_{n}$ are elements of the Lie algebra of $\tilde{G}$ and introduce the mass and gauge parameters in the lower dimensional theory.

Duality symmetries arise naturally in string theory and in supergravity theories. For example, eleven dimensional supergravity compactified on an $n$-torus $T^{n}$ yields in $D=11-$ $n$ dimensions a maximally supersymmetric supergravity theory with the global symmetry group $E_{n, n}[13,14]^{6}$. The $S L(n, \mathbb{R})$ part of the symmetry group $E_{n}$ is obvious and is associated with the large diffeomorphism group of the internal torus $T^{n}$. The symmetry enhancement becomes more clear in string/M theory. The discrete subgroup $E_{n}(Z)$ is the U-duality symmetry of M-theory compactified on $T^{n}$ [10]. The $S L(n, Z)$ part is the geometric part of the T-duality group, whereas the enhancement is associated with the part of the T-duality that mixes momentum and winding modes and the S-duality (which we will refer to as the non-geometric symmetries from here on). As we have reviewed here for $n=2$ case, if the duality twist is in the geometric $S L(n, \mathbb{R})$, then the reduction can be lifted to a string theory compactification on a twisted torus (can be viewed as having arisen as the low energy effective theory limit of such a compactification). On the other hand, when the symmetry is a non-geometric symmetry, then in most cases there is no lifting to

\footnotetext{
${ }^{6}$ Here $E_{n, n}$ is the maximal noncompact form of the exceptional group $E_{n}$. For brevity we write them simply as $E_{n}$. For $n \leq 5$ we have $E_{0}$ trivial, $E_{1}=\mathbb{R}, E_{2}=G L(2, \mathbb{R}), E_{3}=S L(3, \mathbb{R}) \times S L(2, \mathbb{R}), E_{4}=$ $S L(5, \mathbb{R}), E_{5}=O(5,5)$.
} 
a conventional geometric string background [15, 16, 17, 18, 19]. However, the supergravity analysis that we have used here is still valid [2, 20, 16] and one can try and learn from the gauged supergravities arising from dimensional reductions of supergravity theories with general duality twists. This is the approach that has been adopted in the recent paper of Dabholkar and Hull [16]. If the duality twist is through an element of the group that contains and is properly larger than the geometric $S L(n, \mathbb{R})$ subgroup then, from the point of view of the higher dimensional supergravity theory, this amounts to twisting the internal geometry and introducing non-trivial boundary conditions for some of the fields as in (1.2). For this reason, it is important to study duality-twisted reductions on twisted geometries. The analysis that we have presented here is a first step in understanding such dimensional reductions.

As a particular example, we considered here the dimensional reduction of the eight dimensional type II supergravity theory (truncated to the sector of $S L(2, \mathbb{R}$ )-singlets) with an $S L(3, \mathbb{R})$ twist on a three dimensional unimodular, non-semisimple group, which has the local structure of a twisted torus. This eight dimensional theory can be obtained from a $T^{3}$ compactification of the eleven dimensional supergravity and the $S L(3, \mathbb{R})$ symmetry that we exploit is the geometric symmetry associated with the large diffeomorphisms of $T^{3}$. So, the new five dimensional gauged supergravity that we found in section 5 can be viewed as having been obtained from a compactification of M-theory on a non-trivial $T^{3}$ bundle over a three dimensional twisted torus (which itself is a non-trivial $T^{2}$ bundle over $S^{1}$ ). On the other hand, the same eight dimensional theory that we start with can also be obtained as the low energy limit of Type II string theory compactified on a two dimensional torus, $T^{2}$. There is an $S L(2, \mathbb{R})$ symmetry acting on the Kähler modulus of this two-torus (which is the non-geometric part of the T-duality group). It combines with the S-duality $S L(2, \mathbb{R})$ of Type IIB supergravity to form the $S L(3, \mathbb{R})$ symmetry group that we use to introduce a duality-twisted reduction ansatz. So, from the point of view of ten dimensional Type IIB supergravity, the geometry of the five dimensional internal space that leads to the five dimensional gauged supergravity (5.5) is more complicated (and perhaps should be analyzed in the context of Hull's T-folds [15, 21]) as the Kähler modulus of $T^{2}$ varies as it traverses over the three dimensional twisted torus. There are also twisted boundary conditions for higher dimensional fields determined by their transformations under the $S L(2, \mathbb{R})$ S-duality symmetry.

It would be interesting to study the duality-twisted reductions of other supergravity theories on more general twisted geometries and see what this can teach us about string compactifications with U-duality twists. An important step in this direction would be to study the moduli space of flat connections on twisted tori.

\section{Acknowledgements}

I am grateful to Chris Hull for insightful discussions, for reading the manuscript and for useful suggestions. This work is supported by Irish Research Council for Science, Engineering and Technology (IRCSET) under the postdoctoral fellowship scheme. 


\section{Appendix}

\section{A. Dimensional Reduction of $\mathcal{L}_{8}$}

In this appendix we give the details of the dimensional reduction of the eight dimensional theory (5.1) on a twisted torus with a duality twist in $S L(3, \mathbb{R})$.

Let us start by rewriting (2.1), which is the metric ansatz for our dimensional reduction

$$
d \hat{s}^{2}=e^{2 \alpha \phi} d s^{2}+e^{2 \beta \phi} \mathcal{M}_{m n} h^{m} h^{n}
$$

with $h^{m}=\eta^{m}+\mathcal{A}^{m}=\eta^{m}+\mathcal{A}_{\mu}^{m} d x^{\mu}$, where $\eta^{m}$ are the basis one-forms on the three dimensional group manifold which we presented in (4.14), (4.18), (4.22) for the cases of our interest. Above we have split the eight dimensional curved index $\hat{\mu}$ as $\hat{\mu}:(\mu, m)$, where $\mu$ runs from 1 to 5 whereas the internal index $m$ runs from 1 to 3 . There is a corresponding splitting of the flat indices $\hat{a}:(a, i)$. From (A.1) one can find the vielbeins, that is, the basis 1-forms on the flat tangent space:

$$
\begin{gathered}
\hat{e}_{\mu}^{a}=e^{\alpha \phi} e_{\mu}^{a}, \quad \hat{e}_{\mu}^{a}=0, \\
\hat{e}^{i}=e^{\beta \phi} e^{i}=e^{\beta \phi} L_{m}^{i} h^{m},
\end{gathered}
$$

where we have used that $\mathcal{M}_{m n}$ is a symmetric matrix so that it can be written as

$$
\mathcal{M}_{m n}=L_{m}^{i} L_{n}^{j} \delta_{i j}
$$

From (A.2) it follows that

$$
\hat{e}_{\mu}^{i}=e^{\beta \phi} L_{m}^{i} \mathcal{A}_{\mu}^{m}, \quad \hat{e}_{n}^{i}=e^{\beta \phi} e_{n}^{i}=e^{\beta \phi} L_{m}^{i} U_{n}^{m} .
$$

Then we can write

$$
\hat{e}_{\hat{\mu}}{ }^{\hat{a}}=\left(\begin{array}{cc}
e^{-\alpha \phi} e_{\mu}^{a} & e^{\beta \phi} L_{m}^{i} \mathcal{A}_{\mu}^{m} \\
0 & e^{\beta \phi} L_{n}^{i} U_{m}^{n}
\end{array}\right) .
$$

Note that $\alpha$ and $\beta$ can be found from (2.2) by using $D=5, d=3$ to be

$$
\alpha=\frac{1}{2 \sqrt{3}}, \quad \beta=-\alpha=-\frac{1}{2 \sqrt{3}} .
$$

Performing the dimensional reduction on the flat tangent space is standard and is discussed in detail in the literature. We refer the reader to [5, 6] for the reduction of the Einstein-Hilbert term which yields in five dimensions the term (2.5) with $\alpha$ and $\beta$ as in (A.6). Here we will discuss the dimensional reduction of the kinetic terms for the p-form fields and the scalars, for which one also has to consider the new features due to the nontrivial $S L(3, \mathbb{R})$ twist. To analyze this we first need to see how the eight dimensional Hodge operator is related to the five and three dimensional ones. One can show, by using (A.2) and (A.4) that

$$
\begin{aligned}
\hat{*}\left(X_{(p) m_{1} \cdots m_{r}} \frac{h^{m_{1}} \wedge \cdots \wedge h^{m_{r}}}{r !}\right) & =e^{-p \alpha \phi} e^{-r \beta \phi} e^{(D-p) \alpha \phi} e^{(d-r) \beta \phi} *_{D}\left(X_{(p)}\right) *_{d}\left(\frac{h^{m_{1}} \wedge \cdots \wedge h^{m_{r}}}{r !}\right) \\
& =e^{(1-p+r) / \sqrt{3} \phi_{*_{5}}\left(X_{(p)}\right) *_{3}\left(\frac{h^{m_{1}} \wedge \cdots \wedge h^{m_{r}}}{r !}\right) .}
\end{aligned}
$$


In the last line we have used that $D=5, d=3$ and $\alpha$ and $\beta$ are as in (A.6). Then we have

$$
\begin{aligned}
\hat{*}_{8} \hat{H}_{(3)}= & \hat{*}_{8}\left[H_{(3)}+H_{(2) n} \wedge h^{n}+\frac{1}{2 !} \epsilon_{m n p} H_{(1)}^{m} \wedge h^{n} \wedge h^{p}+\frac{1}{3 !} \epsilon_{m n p} H_{(0)} h^{m} \wedge h^{n} \wedge h^{p}\right] \\
= & e^{-4 \alpha \phi} *_{5} H_{(3)} *_{3} 1+*_{5} H_{(2) n} *_{3} h^{n}+e^{2 \alpha \phi} \epsilon_{m n p} *_{5} H_{(1)}^{m} *_{3}\left(\frac{h^{n} \wedge h^{p}}{2 !}\right) \\
& +e^{8 \alpha \phi} *_{5} H_{(0)} *_{3}\left(\epsilon_{m n p} \frac{h^{m} \wedge h^{n} \wedge h^{p}}{3 !}\right) .
\end{aligned}
$$

From (A.8) we can easily deduce that the dimensional reduction of $\hat{H}_{(3)}^{t} \hat{\mathcal{M}}^{-1} \hat{*}_{8} \hat{H}_{(3)}$ yields (5.9) by noticing a few important points we list below.

$$
\begin{aligned}
& h^{m} \wedge *_{3} h^{n}=L_{i}^{m} L_{j}^{n} e^{i} \wedge *_{3} e^{j}=L_{i}^{m} L_{j}^{n} \delta^{i j}=\mathcal{M}^{m n} *_{3} 1 . \\
& \left(\frac{1}{3 !} \epsilon_{m n p} h^{m} \wedge h^{n} \wedge h^{p}\right) \wedge *_{3}\left(\frac{1}{3 !} \epsilon_{q r s} h^{q} \wedge h^{r} \wedge h^{s}\right)=*_{3} 1 . \\
& \epsilon_{m p q} \epsilon_{n r s}\left(\frac{h^{p} \wedge h^{q}}{2 !}\right) \wedge *_{3}\left(\frac{h^{r} \wedge h^{s}}{2 !}\right)=\epsilon_{i_{1} i_{2} i_{3}} \epsilon_{j_{1} j_{2} j_{3}}\left(\frac{e^{i_{2}} \wedge e^{i_{3}}}{2 !}\right) \wedge *_{3}\left(\frac{e^{j_{2}} \wedge e^{j_{3}}}{2 !}\right) \\
& =\epsilon_{i_{1} i_{2} i_{3}} \epsilon_{j_{1} j_{2} j_{3}} \frac{\left(\delta^{i_{2} j_{2}} \delta^{i_{3} j_{3}}-\delta^{i_{3} j_{2}} \delta^{i_{2} j_{3}}\right)}{4} *_{3} 1=\frac{\epsilon_{i_{1} j_{2} j_{3}} \epsilon_{j_{1} j_{2} j_{3}}}{2} *_{3} 1 \\
& =\frac{\left(\delta_{j_{2} j_{2}} \delta_{i_{1} j_{1}}-\delta_{j_{2} i_{1}} \delta_{j_{1} j_{2}}\right)}{2} *_{3} 1=\delta_{i_{1} j_{1}} \text {. }
\end{aligned}
$$

Now consider the dimensional reduction for the kinetic term (3.8) for the scalar fields. Following (3.9) we find

$$
\begin{aligned}
d \hat{\mathcal{K}} & =\tilde{g}\left(M_{p} \mathcal{K} \eta^{p}+d \mathcal{K}+\mathcal{K} M_{p}^{t} \eta^{p}\right) \tilde{g}^{t} \\
& =\tilde{g}\left(M_{p} \mathcal{K} h^{p}-M_{p} \mathcal{K} \mathcal{A}^{p}+d \mathcal{K}+\mathcal{K} M_{p}^{t} h^{p}-\mathcal{K} M_{p}^{t} \mathcal{A}^{p}\right) \tilde{g}^{t} \\
& =\tilde{g}\left(M_{i} \mathcal{K} e^{i}-M_{p} \mathcal{K} \mathcal{A}^{p}+d \mathcal{K}+\mathcal{K} M_{i}^{t} e^{i}-\mathcal{K} M_{p}^{t} \mathcal{A}^{p}\right) \tilde{g}^{t}
\end{aligned}
$$

where $M_{i}=L_{i}{ }^{m} M_{m}$. (Remember that $e^{i}=L_{m}^{i} h^{m}$.) Similarly,

$$
\begin{aligned}
d \hat{\mathcal{K}}^{-1} & =\left(\tilde{g}^{t}\right)^{-1}\left(-M_{p}^{t} \mathcal{K}^{-1} \eta^{p}+d \mathcal{K}^{-1}-\mathcal{K}^{-1} M_{p} \eta^{p}\right) \tilde{g}^{-1} \\
& =\left(\tilde{g}^{t}\right)^{-1}\left(-M_{p}^{t} \mathcal{K}^{-1} h^{p}+M_{p}^{t} \mathcal{K}^{-1} \mathcal{A}^{p}+d \mathcal{K}^{-1}-\mathcal{K}^{-1} M_{p} h^{p}+\mathcal{K}^{-1} M_{p} \mathcal{A}^{p}\right) \tilde{g}^{-1} \\
& =\left(\tilde{g}^{t}\right)^{-1}\left(-M_{i}^{t} \mathcal{K}^{-1} e^{i}+M_{p}^{t} \mathcal{K}^{-1} \mathcal{A}^{p}+d \mathcal{K}^{-1}-\mathcal{K}^{-1} M_{i} e^{i}+\mathcal{K}^{-1} M_{p} \mathcal{A}^{p}\right) \tilde{g}^{-1}
\end{aligned}
$$

From (A.10) and (A.11) we have

$\operatorname{tr}\left(d \hat{\mathcal{K}} \wedge \hat{*} d \hat{\mathcal{K}}^{-1}\right)=\operatorname{tr}\left[\tilde{g}\left(D \mathcal{K}+\left(M_{i} \mathcal{K}+\mathcal{K} M_{i}^{t}\right) e^{i}\right) \tilde{g}^{t} \wedge *_{3}\left(\tilde{g}^{t}\right)^{-1}\left(D \mathcal{K}^{-1}-\left(M_{i}^{t} \mathcal{K}^{-1}+\mathcal{K}^{-1} M_{i}\right) e^{i}\right) \tilde{g}^{-1}\right]$,

where $D \mathcal{K}$ and $D \mathcal{K}^{-1}$ are as in (5.7). From (A.12) we find (5.6) by using (A.7) and $e^{i} \wedge *_{3} e^{j}=\delta^{i j} *_{3} 1$.

Let us now look at the dimensional reduction of the topological term

$$
\epsilon^{a b c} \hat{H}_{(3) a} \wedge \hat{H}_{(3) b} \wedge \hat{B}_{(2) c},
$$


where $\hat{B}_{(2)}$ and $\hat{H}_{(3)}$ are as in (3.14) and 3.15$)$, respectively. Note that the $y$ dependence introduced through $\tilde{g}(y)$ cancels out in five dimensions as we have

$$
\epsilon^{a b c} S_{a}{ }^{d} S_{b}{ }^{e} S_{c}{ }^{f}=\epsilon^{d e f},
$$

for all $S \in S L(3)$. The term (A.13) does not involve the metric, so its dimensional reduction is straightforward. The important point we should note is that the only non-zero terms are the ones of the form

$$
\sim \epsilon H_{(i)} \wedge H_{(j)} \wedge B_{(k)} \wedge h \wedge h \wedge h
$$

with $i+j+k=5$ as the dimensions of the internal and the base space are three and five respectively. From the product (A.13) one obtains nine such terms some of which cancel each other. It is easy to check that the remaining terms give (5.10).

\section{References}

[1] J. Scherk and J. H. Schwarz, "Spontaneous Breaking Of Supersymmetry Through Dimensional Reduction," Phys. Lett. B 82 (1979) 60.

[2] A. Dabholkar and C. Hull, "Duality twists, orbifolds, and fluxes," JHEP 0309 (2003) 054 [arXiv:hep-th/0210209].

[3] J. Scherk and J. H. Schwarz, "How to get masses from extra dimensions", Nucl. Phys. B 153, (1979) 61 .

[4] C. M. Hull and R. A. Reid-Edwards, "Flux compactifications of string theory on twisted tori," arXiv:hep-th/0503114.

[5] M. Cvetic, G. W. Gibbons, H. Lu and C. N. Pope, "Consistent group and coset reductions of the bosonic string," arXiv:hep-th/0306043.

[6] D. Roest, "M-theory and gauged supergravities," Fortsch. Phys. 53 (2005) 119 [arXiv:hep-th/0408175].

[7] G. Dall'Agata and S. Ferrara, "Gauged supergravity algebras from twisted tori compactifications with fluxes," Nucl. Phys. B 717 (2005) 223 [arXiv:hep-th/0502066].

[8] N. Kaloper and R. C. Myers, "The O(dd) story of massive supergravity," JHEP 9905, (1999) 010 [arXiv:hep-th/9901045].

[9] E. Bergshoeff, U. Gran, R. Linares, M. Nielsen, T. Ortin and D. Roest, "The Bianchi classification of maximal D = 8 gauged supergravities," Class. Quant. Grav. 20 (2003) 3997 [arXiv:hep-th/0306179].

[10] C. M. Hull and P. K. Townsend, "Unity of superstring dualities," Nucl. Phys. B 438 (1995) 109 [arXiv:hep-th/9410167].

[11] A. Salam and E. Sezgin, "D = 8 Supergravity," Nucl. Phys. B 258 (1985) 284.

[12] N. Alonso-Alberca, P. Meessen and T. Ortin, "An SL(3,Z) multiplet of 8-dimensional type II supergravity theories and the gauged supergravity inside," Nucl. Phys. B 602 (2001) 329 [arXiv:hep-th/0012032]. 
[13] E. Cremmer, "Dimensional Reduction In Field Theory And Hidden Symmetries In Extended Supergravity," LPTENS 81/18 Lectures given at ICTP Spring School Supergravity, Trieste, Italy, Apr 22 - May 6, 1981

[14] E. Cremmer, B. Julia, H. Lu and C. N. Pope, "Dualisation of dualities. I," Nucl. Phys. B 523 (1998) 73 [arXiv:hep-th/9710119].

[15] C. M. Hull, "A geometry for non-geometric string backgrounds," JHEP 0510 (2005) 065 [arXiv:hep-th/0406102].

[16] A. Dabholkar and C. Hull, "Generalised T-duality and non-geometric backgrounds," JHEP 0605 (2006) 009 [arXiv:hep-th/0512005].

[17] A. Flournoy, B. Wecht and B. Williams, "Constructing nongeometric vacua in string theory," Nucl. Phys. B 706 (2005) 127 [arXiv:hep-th/0404217].

[18] J. Shelton, W. Taylor and B. Wecht, "Nongeometric flux compactifications," JHEP 0510 (2005) 085 [arXiv:hep-th/0508133].

[19] A. Flournoy and B. Williams, "Nongeometry, duality twists, and the worldsheet," JHEP 0601 (2006) 166 [arXiv:hep-th/0511126].

[20] C. M. Hull and A. Catal-Ozer, "Compactifications with S-duality twists," JHEP 0310 (2003) 034 [arXiv:hep-th/0308133].

[21] C. M. Hull, "Doubled geometry and T-folds," arXiv:hep-th/0605149. 